\title{
EXPERIMENTAL EVALUATION OF STEREOLOGICAL METHODS FOR DETERMINING 3D GRAIN SIZE AND TOPOLOGICAL DISTRIBUTIONS
}

\author{
GUOQUAN LIU, HAIBO YU \\ University of Science and Technology Beijing, 100083 Beijing, China \\ E-mail: gq_liu@yahoo.com or G.Liu@ustb.edu.cn \\ (Accepted April 25, 2000)
}

\begin{abstract}
The conventional serial sectioning analysis and a set of modern stereological methods, including disector, selector, point-sampled intercepts, point-sampled area, and their combinations, have been used in this paper to measure the grain size, grain size distribution, topological parameters and their distributions in a spacefilling single-phase grain structure of steel. The results from different methods are compared and used to evaluate the methods quantitatively, based on which some suggestions will be given for selection of experimental methods in materials stereology research.
\end{abstract}

Keywords: 3D microstructure, space-filling grains, stereology, disector, selector, point-sampled intercepts, point sampled area, serial sectioning, grain size distribution, grain topology.

\section{INTRODUCTION}

There have been significant advances in both theoretical and practical stereology especially since Sterio (1984) proposed to use disector for unbiased estimation of number and size of arbitrary particles in $3 \mathrm{D}$ space. By centering on the design of sampling strategies, the disector method first dropped all inherent assumptions about the particle shape in previous analysis methods, then the point-sampled intercept and some other methods followed. Several related monographs related can be easily found elsewhere (e.g., Cruz-Orive and Weibel, 1990; Weibel, 1992; Liu, 1993; Gokhale and Deshpande, 1994; Kurzydlowski and Ralph, 1995; Howard and Reed, 1998).

However, applications of these new methods in materials science and engineering are not common. Srinivasan et al. (1991 and 1992), Kurzydlowski and Bucki (1992) used the point-sampled intercept method to estimate volume-weighted grain size and grain size uniformity in polycrystalline materials. Vonder Voort and Gokhale (1992) also discussed the point-sampled intercept issue. However, the materials scientists do not prefer to use volume-weighted distribution to characterize microstructures.

The disector method does provide valuable information of number-weighted means of grain size and topology. Karlsson and Cruz-Orive (1992) first used the disector to evaluate the size and numbers of discrete pores existing in aluminum. In order to determine the size and number of space-filling grains in materials or space-filling cells in biology, Liu et al. (1994) modified the counting rules of the disector for discrete particles. They further combined their modified disector analysis with the point-sampled intercept method to obtain more quantitative information on both the number-weighted and the volume-weighted size distributions for space-filling grains. Yu et al. (1998) further demonstrated the reliability and objectivity of the point-sampled intercept method, the point-sampled area method and the disector method when applied to space-filling polycrystalline materials, and proposed methods for converting the volume-weighted distribution data into number-weighted ones.

As mentioned above, the new group of designbased stereology methods has been found useful for microstructure quantification at limited levels. It seems that the conventional serial sectioning analysis may still be necessary if a more detail quantitative description of the microstructure is required (as summarized in Table 1). It is the purpose of this work to give a critical review and experimental assessment on the applicability and the objectivity of these unbiased design-based stereology methods for spacefilling polycrystalline structures by applying simultaneously all these methods to the same specimen. 


\section{MATERIALS AND EXPERIMENTAL METHODS}

The specimens analyzed were the same as the samples 3S and 3M used by $\mathrm{Yu}$ et al. (1998), and were prepared by standard metallographic procedures. Microscopic examination on three mutually perpendicular surfaces of the samples showed that the microstructures are essentially isotropic and that the grains are nearly equiaxed. Conventional planar metallographic analysis revealed that the mean linear intercept grain sizes for the $3 \mathrm{~S}$ and $3 \mathrm{M}$ samples are 32 and $42 \mu \mathrm{m}$, respectively. The sample $3 \mathrm{~S}$ (Fig. 1) is used here to get a general idea about the applicability of different stereology methods including serial sectioning analysis, while the latter for modern unbiased design-based methods only.

By obtaining 100 sections about $3 \mu \mathrm{m}$ apart, totally 1292 contiguous grains in the sample $3 \mathrm{~S}$ were individually measured by using conventional serial sectioning method. The parameters measured include:
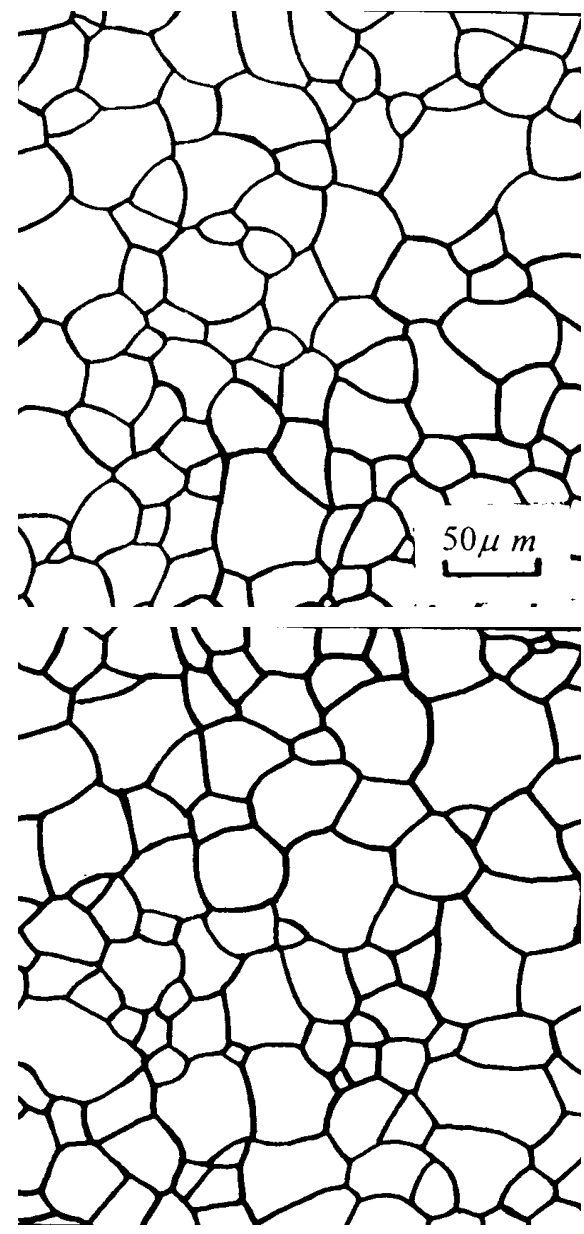

The volume $V$ and the number of faces $F$, edges $E$, and corners $C$ of individual 3D grains and their corresponding means such as $\overline{v_{N}}$ (number-weighted) and $\overline{v_{V}}$ (volume-weighted), and $\bar{F}, \bar{E}, \bar{C}$;

The number of grains per specimen volume $N_{V}$; the number of grain faces $F_{V}$, edges $E_{V}$, and corners $C_{V}$ per volume;

The coefficient of variation $C V_{N}(v)$, the kurtosis $C E_{N}(v)$, and the skewness $C S_{N}(v)$ of the numberweighted grain volume distribution;

The coefficient of variation $C V_{V}(v)$, the kurtosis $C E_{V}(v)$, and the skewness $C S_{V}(v)$ of the volumeweighted grain volume distribution;

The coefficient of variation $C V_{N}(F)$, the kurtosis $C E_{N}(F)$, and the skewness $C S_{N}(F)$ of the numberweighted grain face number distribution; and so on.

The number-weighted mean number of sides of grain faces in 3D space, $\bar{n}$.
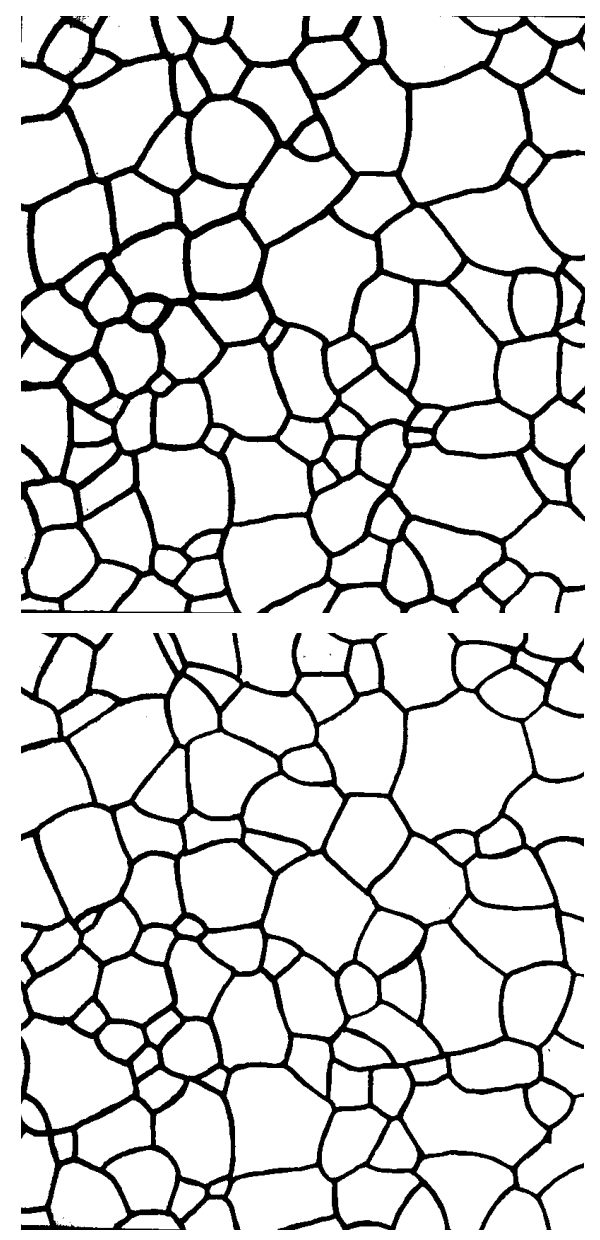

Fig. 1. Four serial sections of the single-phased polycrystalline sample $3 S$ of steel analysed. 
It has been experimentally proved that such amount of sampled grains is adequate to obtain reliable information on grain volume distribution and to estimate grain topology distribution ( $\mathrm{Liu}$ and $\mathrm{Yu}$, 1999).

The detailed procedure of disector method applied here is the same as proposed by Liu et al. (1994). To ensure statistically representative results, at least 2000 grain transects in each sample were sampled by the unbiased counting frame on one section and checked for their appearance on another section, and the total volume sampled was about $8 \times 10^{-11} \mathrm{~m}^{3}$ per sample. The parameters measured include: $C_{V}, E_{V}, F_{V}, N_{V}, \bar{C}, \bar{E}, \bar{F}$ and $\overline{v_{N}}$.

The point-sampled intercept method was used here to estimate the volume-weighted grain volume, $\overline{v_{V}}$, as proposed by Gundersen and Jensen (1985). It is an absolute requirement for the estimation that the point-sampled intercept lengths are measured in an isotropic uniform random direction with respect to the grains. Combined with the disector method, the coefficient of variation of the number-weighted grain volume distribution was obtained:

$$
C V_{N}(v)=\left[\left(\overline{v_{V}} / \overline{v_{N}}\right)-1\right]^{1 / 2} .
$$

When the point-sampled area method was used here to estimate the $2^{\text {nd }}$ moment of the volumeweighted grain volume distribution, $\overline{v_{V}^{2}}$, the following relationship was applied (Jensen and Sorensen, 1991):

$$
\overline{v_{V}^{2}}=4 \pi \mathrm{C} \overline{a_{0}^{3}}
$$

where $\overline{a_{0}^{3}}$ denotes the mean value of $3^{\text {rd }}$ power of the area of point-sampled grains over the sample analyzed, $C$ was taken as 0.075 for a first approximation.

\begin{tabular}{|c|c|c|c|c|}
\hline \multirow[t]{2}{*}{ Method } & \multicolumn{4}{|c|}{ Possible parameters able to be evaluated } \\
\hline & $\begin{array}{l}\text { Mean size } \\
\text { parameters }\end{array}$ & $\begin{array}{l}\text { Volume } \\
\text { distribution } \\
\text { parameters }\end{array}$ & $\begin{array}{l}\text { Mean topological } \\
\text { parameters }\end{array}$ & $\begin{array}{l}\text { Topological } \\
\text { distribution } \\
\text { parameters }\end{array}$ \\
\hline (1) Serial sectioning method ${ }^{\mathrm{a}}$ & $\frac{\overline{v_{N}}}{\overline{v_{V}^{n}}}, \overline{\overline{v_{V}}}$, & $\begin{array}{l}C V_{N}(v), C V_{V}(v), \\
C S_{N}(v), C S_{V}(v), \\
C E_{N}(v), C E_{V}(v)\end{array}$ & 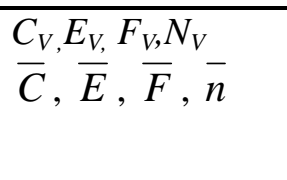 & $\begin{array}{l}C V_{N}(F) \\
C S_{N}(F) \\
C E_{N}(F)\end{array}$ \\
\hline (2) Disector method & $\overline{v_{N}}$ & & 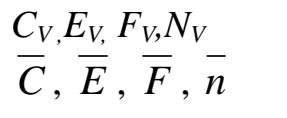 & \\
\hline (3) Point- sampled intercepts & $\overline{v_{V}}$ & & & \\
\hline (4) Point- sampled areas & $\overline{v_{V}^{2}}$ & & & \\
\hline (5) Selector method & $\overline{v_{N}}$ & & 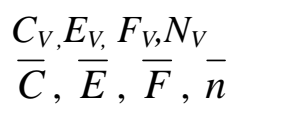 & \\
\hline$(2)+(3)$ & & $C V_{N}(v)$ & & \\
\hline$(5)+(3)$ & & $C V_{N}(v)$ & & \\
\hline$(3)+(4)$ & & $C V_{V}(v)$ & & \\
\hline$(2)+(3)+(4)$ & & $C S_{N}(v)$ & & \\
\hline$(5)+(3)+(4)$ & & $C S_{N}(v)$ & & \\
\hline
\end{tabular}

Table 1. Characteristic parameters measured by different stereological methods. 
Combining the point-sampled area and the pointsampled intercept methods, we obtained the coefficient of variation of the volume-weighted grain volume distribution:

$$
C V_{V}(v)=\frac{\sqrt{\overline{v_{V}^{2}}-{\overline{v_{V}}}^{2}}}{\overline{v_{V}}} .
$$

By applying the disector, the point-sampled intercept and the point-sampled area methods to get $\overline{v_{N}}, \overline{v_{V}}$, and $\overline{v_{V}^{2}}$, respectively, we calculated the skewness of the number-weighted grain volume distribution without applying serial sectioning analysis, according to the following equation (Gundersen and Jensen 1985):

$$
C S_{N}(v)=\frac{\overline{v_{V}^{2}}-3 \overline{\nu_{N}} \cdot \overline{v_{V}}+2{\overline{\nu_{V}}}^{2}}{{\overline{v_{N}}}^{\frac{1}{2}}\left(\overline{\nu_{V}}-\overline{v_{N}}\right)^{\frac{3}{2}}} .
$$

The selector method proposed by Cruz-Orive (1987) was also used in this work for the estimation of $\overline{v_{N}}$. Firstly, certain amount of grains is sampled with identical probabilities using a disector of unknown thickness. Secondly, the volume of each sampled grain is unbiased estimated by means of the point-sampled intercept method on their grain transects.

\section{RESULTS AND DISCUSSION}

All the experimental results obtained are summarized in Tables 2, 3, and 4, as well as in Fig. 2.

One can quantify the microstructure at different levels with different combinations of information and data. For example, the quantification at different metric quantification levels; or, the quantification of both the metric and topological global and mean properties; or, the quantification of distributions of grain size and topology, with both the values of characterization parameters and the plots of the whole frequency distribution curves shown.

For example, Table 2 lists the results of the number-weighted mean grain volume $\overline{v_{N}}$ and those mean topological parameters $\bar{C}, \bar{E}, \bar{F}, \bar{n}$, along with the global topological parameters $N_{V}, C_{V}, E_{V}$, and $F_{V}$ for the sample $3 \mathrm{~S}$. Obviously, both the disector and the serial sectioning methods can estimate these parameters well. If one needs to quantify the microstructure at this level with such a combination of parameters, the disector method is already adequate. Compared with the serial sectioning analysis, the disector improves the efficiency significantly. One reason is that the number of the dependent section planes needed has been significantly reduced, another is that it is no longer necessary to give an individual identification number to each grain as in the serial sectioning analysis. Another main advantage of the disector is that nothing is assumed about the grains to be counted except the fulfilling of a general requirement, i.e. it is possible to unambiguously identify different isolated grain sections belonging to the same grain on the section plane.

When a much more detailed quantification of the grain volume distributions is required, as summarized in the first line of Table 3 , the serial sectioning analysis is indispensable, since the disector method tells nothing about the distribution of grain volumes or grain topology. To date, no single modern unbiased stereology method, or even a combination of such methods, is found able to provide simultaneously all the four basic parameters of the number-weighted grain volume distribution: $\overline{v_{V}}$, $C V_{N}(v), C E_{N}(v)$, and $C S_{N}(v)$. This is also true for the distributions of topological parameters of grains, or for the volume-weighted grain volume distribution. The serial sectioning method does the jobs well, with the frequency distribution curves also available, as plotted for the steel sample 3S in Fig. 2.

Table 2. Selected experimental results of the sample $3 S$ by the disector and the serial sectioning.

\begin{tabular}{llllllllll}
\hline & $\mathrm{N}_{\mathrm{V}}$, & $\overline{V_{N}}$, & $C_{V}$, & $E_{V}$, & $F_{V}$, & $\bar{C}$ & $\bar{E}$ & $\bar{F}$ & - \\
& $10^{9} \mathrm{~m}^{-3}$ & $10^{-12} \mathrm{~m}^{3}$ & $10^{9} \mathrm{~m}^{-3}$ & $10^{9} \mathrm{~m}^{-3}$ & $10^{9} \mathrm{~m}^{-3}$ & & & & \\
& 15100 & 0.0662 & 79932 & 15986 & 95042 & 21.15 & 31.73 & 12.58 & 5.046 \\
Disector method & & & & & & & & & \\
Serial sectioning analysis & 14800 & 0.0678 & 80314 & 16028 & 95064 & 21.78 & 32.67 & 12.89 & 5.069 \\
\hline
\end{tabular}


Except for the kurtosis of the distribution, it is shown in Tables 3 and 4 that almost all the other parameters can be obtained by modern design-based stereology methods. It can be seen in Table 3 that the estimates of all parameters obtained by using the disector, the selector, the point-sampled intercepts, and the point-sampled areas or their combinations, are all in good agreement with those obtained by the serial sectioning analysis. Therefore, both the reliability and the precision of such measurements in polycrystalline materials have been illustrated.

The results show that $\overline{V_{V}}$ is always larger than $\overline{V_{N}}$ for a given sample (Tables 3 and 4 ); it is inappropriate to use $\overline{V_{V}}$ as an approximation of $\overline{v_{N}}$.
The relative frequency distributions of the volume and the volume-equivalent sphere diameter of the 1292 grains in the sample $3 \mathrm{~S}$ are shown in Figs. $2 \mathrm{a}$ and $\mathrm{b}$, and the corresponding distribution of number of grain faces in Fig. 1d. All of them were determined by serial sectioning analysis. It can be seen from Figs. $2 \mathrm{a}$ and $2 \mathrm{~b}$ that the grain size distributions are very typical as often found in materials science, and are better fitted to gamma distribution, rather than a lognormal distribution function. Fig. $2 \mathrm{c}$ also illustrates that the grain volume data do not fit the lognormal function well. This conclusion has been further supported by theoretical model-based calculations (Yu et al., 1998).

Table 3. Experimental results of grain volume distribution parameters for the steel sample $3 S$.

\begin{tabular}{|c|c|c|c|c|c|c|c|c|}
\hline & $\begin{array}{l}\mathrm{N}_{\mathrm{V}} \\
10^{9} \mathrm{~m}^{-3}\end{array}$ & $\begin{array}{l}\overline{v_{N}} \\
10^{-14} \mathrm{~m}^{3}\end{array}$ & $\begin{array}{l}\overline{v_{V}} \\
10^{-13} \mathrm{~m}^{3}\end{array}$ & $\begin{array}{l}\overline{v_{V}^{2}} \\
10^{-26} \mathrm{~m}^{6}\end{array}$ & $C V_{N}(v)$ & $C V_{V}(v)$ & $C E_{N}(v)$ & $C S_{N}(v)$ \\
\hline $\begin{array}{l}\text { (1) Serial Sectioning } \\
\text { analysis }\end{array}$ & 14800 & 6.78 & 1.5 & 3.6 & 1.14 & 0.774 & 7.465 & 2.296 \\
\hline (2) Disector method & 15100 & 6.62 & & & & & & \\
\hline (3) Point-sampled intercept & & & 1.4 & & & & & \\
\hline (4) Point-sampled area & & & & 3.1 & & & & \\
\hline (5) Selector method & 14600 & 6.85 & & & & & & \\
\hline$(2)+(3)$ & & & & & 1.06 & & & \\
\hline$(5)+(3)$ & & & & & 1.02 & & & \\
\hline$(3)+(4)$ & & & & & & 0.763 & & \\
\hline$(2)+(3)+(4)$ & & & & & & & & 2.319 \\
\hline$(5)+(3)+(4)$ & & & & & & & & 2.321 \\
\hline
\end{tabular}

Table 4. Experimental results of grain volume distribution parameters for the steel sample $4 S$.

\begin{tabular}{|c|c|c|c|c|c|c|}
\hline & $\begin{array}{l}\overline{v_{N}} \\
10^{-13} \mathrm{~m}^{3}\end{array}$ & $\begin{array}{l}\overline{v_{V}} \\
10^{-13} \mathrm{~m}^{3}\end{array}$ & $\begin{array}{l}\overline{v_{V}^{2}} \\
10^{-26} \mathrm{~m}^{6}\end{array}$ & $C V_{N}(v)$ & $C V_{V}(v)$ & $C S_{N}(v)$ \\
\hline (1) Disector method & 1.253 & & & & & \\
\hline (2) Point-sampled intercept method & & 2.730 & & & & \\
\hline (3) Point-sampled area method & & & 11.9 & & & \\
\hline$(1)+(2)$ & & & & 1.09 & & \\
\hline$(2)+(3)$ & & & & & 0.72 & \\
\hline$(1)+(2)+(3)$ & & & & & & 2.378 \\
\hline
\end{tabular}


According to our experimental experience, the following considerations are important for the valid application of modern design-based methods concerned in this paper and for the objectivity of the experimental results in polycrystalline materials:

1. For valid application of such methods, it must be possible to identify possible different isolated transects belonging to the same grain on the section plane. Otherwise, systematic errors will be introduced. For example, possible multiprofile appearance of a grain in a section plane due to the concavity of the grain surface will lead to an underestimation of $\overline{v_{N}}$ by using the disector and $\overline{V_{V}}$ by using the point-sampled intercepts. However, it is found that the possibility that for an actual grain yielding more than one grain transects on a single metallographic plane is very low. Only 21 multi-profile transects belonging to 8 grains among almost 2000 grain transects were found in the serial sectioning analysis in this work. So it can be concluded that for the spacefilling grain structures investigated, this type of systematic error may be negligible.

2. Estimating the number-weighted mean grain
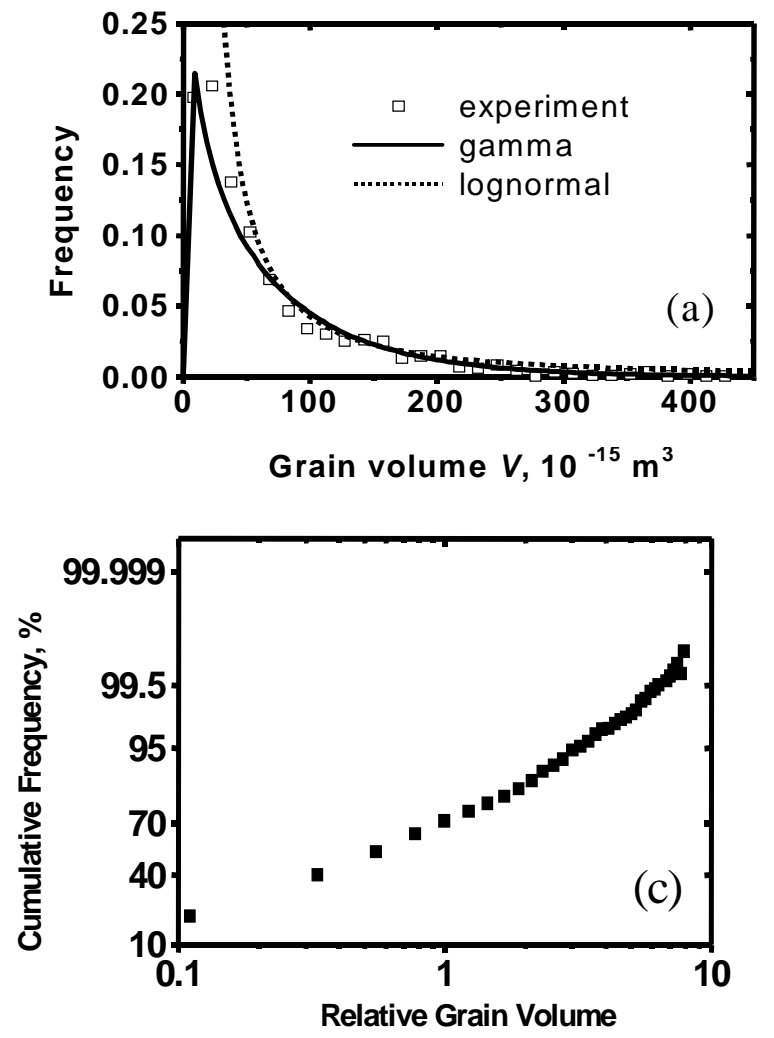

volume requires using three dimensional probes. If the distance between its two planes can be assessed accurately, then the disector method would be an excellent choice. Otherwise, the selector method is suggested. Not having to know the distance between section planes is an invaluable advantage of the selector over the disector.

3. Some of the volume-weighted grain volume distribution parameters, such as $\overline{v_{V}}, \overline{v_{V}^{2}}$ and $C N_{V}(v)$, can be estimated on single, independent sections using the point-sampled intercept and the point-sampled area methods. Furthermore, the measurement can be conducted with high precision and automated by the use of automatic image analysis systems. Such kinds of data, however, are not preferred by materials scientists and engineers. Their usage is to be converted into number-weighted data, either using a combination of different unbiased methods as in this work, or using model-based converting methods (for examples, see Yu et al., 1998).

Finally it may be worth pointing out that the disector and the serial sectioning analysis require only uniform sampling of probes. The other methods, however, require isotropy in addition.
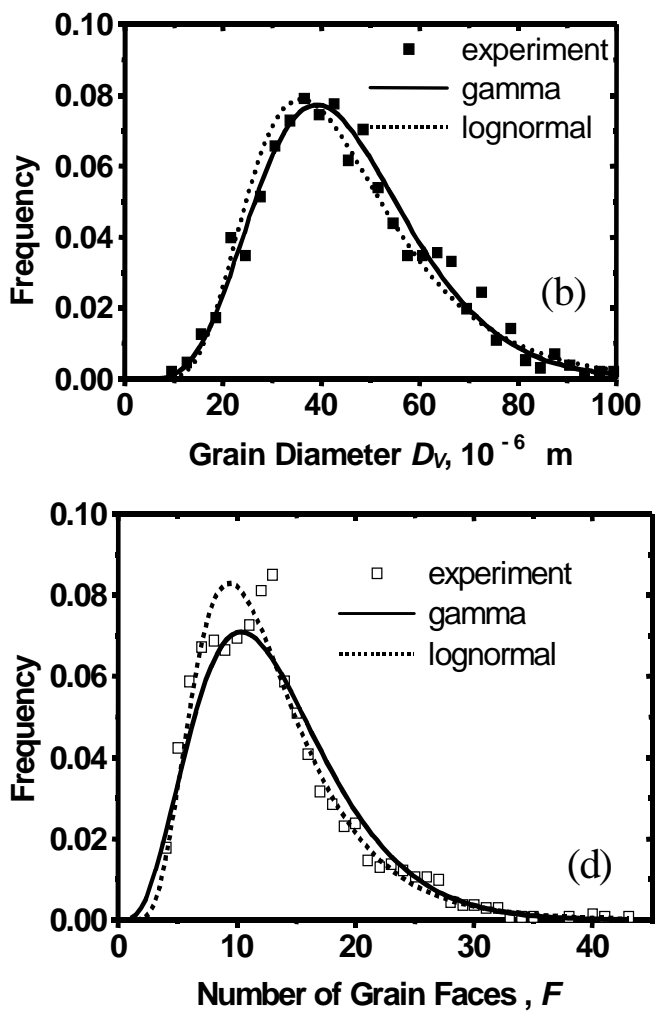

Fig. 2. Relative frequency distributions of grain volume (a) and volume-equivalent sphere diameter (b), cumulative frequency distribution of grain volume (c), and relative frequency of distribution of number of faces (d) of 1292 austenite grains in sample 3S, experimentally determined by serial sectioning analysis. 


\section{CONCLUSIONS}

1. Selected modern design-based stereology methods, including the disector, the selector, the point-sampled intercept, the point-sampled area methods and some of their combinations, were applied simultaneously to single-phased polycrystalline steel samples. The experimental results show that all the estimates obtained by using such techniques are in good agreement with those obtained by using the serial sectioning analysis. The reliability and the precision of such measurements in polycrystalline materials has been illustrated.

2. When a much more detailed quantification of the grain volume or topology distributions is required, especially when the plots of the frequency distributions are required, the serial sectioning analysis is still an indispensable tool.

3. The experimental results show that the numberweighted grain size distributions are better fitted to gamma distribution functions, rather than lognormal ones.

4. For different purposes in different research fields, the microstructure needs to be characterized quantitatively at different levels with different combinations of information and data. Even the so-called "simple, efficient" method consumes time and precious manpower. Never overuse the stereology methods when they are not extremely necessary.

A preliminary report of some of the data was presented at the Xth International Congress for Stereology, Melbourne, Australia, 1-4 November 1999.

\section{ACKNOWLEDGMENTS}

This work was supported by research grants from the National Natural Science Foundation of China (Projects 59872003, 59771070, and 59271063) and grants from the Ministry of Education of China.

\section{REFERENCES}

Cruz-Orive LM (1987). Particle number can be estimated using a disector of unknown thickness: the selector. $\mathbf{J}$ Microsc 145(2):121-42.
Cruz-Orive LM, Weibel ER (1990). Recent stereological methods for cell biology: a brief survey. Am J Physiol 258:L148-56.

Gokhale AM, Deshpande NU (1994). Applications of stereology in materials science and engineering. Acta Stereol 13(2):253-67.

Gundersen HJG, Jensen EB (1985). Stereological estimation of the volume-weighted mean volume of arbitrary particles on random sections. J Microsc 138(12):127-42.

Howard CV, Reed MG (1998). Unbiased stereology three-dimensional measurement in microscopy. Oxford: BIOS Scientific Publishers.

Jensen EB, Sorensen FB (1991). A note on stereological estimation of the volume-weighted second moment of particle volume. J Microsc 164(1):21-7.

Karlsson LM, Cruz-Orive LM (1992). The new stereological tools in metallography: estimation of pore size and number in aluminum. $J$ Microsc 165:391-415.

Kurzydlowski KJ, Ralph B (1995). The quantitative description of the microstructure of materials. New York, London, Tokyo: CRC Press.

Kurzydlowski KJ, Bucki JJ (1992). A method for grain size and grain size uniformity estimation - applications to polycrystalline materials. Scripta Matell Mater 27:117-20.

Liu G (1993). Invited review: applied stereology in materials science and engineering. $\mathrm{J}$ Microsc 171(pt.1):57-68.

Liu G, Yu H (1999). On the sampling of serial sectioning technique for three dimensional space-filling grain structures. Presented at the Xth International Congress for Stereology. Melbourne, Australia; $1^{\text {st }}-4^{\text {th }}$ November.

Liu G, Yu H, Li W (1994). Efficient and unbiased evaluation of grain size and topology. Acta Stereol 13:281-6.

Srinivasan S, Russ JC, Scattergood RO (1992). Reply to "Grain size measurements using the point-sampled intercept technique." Scripta Metall Mater 26:1661-2.

Vonder Voort GF, Gokhale AM (1992). Comments on "Grain size measurements using the point-sampled intercept technique." Scripta Metall Mater 26:1665.

Weibel ER (1992). Stereology in perspective: a mature science evolves. Acta Stereol 11/Suppl 1:1-13.

Yu H, Liu G, Song X (1998). On the relation between the number-weighted and volume-weighted grain volume distribution parameters. Metall Mater Trans A 29A(12):3081. 